\title{
LA ROTTA NIGERIANA DEL TRAFFICO DI RIFIUTI TOSSICI ITALIANI (1987-1988)
}

\author{
Andrea Carnì
}

Title: The nigerian route of italian toxic waste traffic (1987-1988)

\begin{abstract}
The article focuses on the international traffic of Italian hazardous waste directed to Nigeria between 1987 and 1988. In particular it analyses the Italian-Nigerian route, which was the one most used by the companies that organized and carried out the traffic during the 1980s, the actors who took part in it, the goods that were unloaded and the important - and so far scarcely explored - effects on the bilateral relations between Italy and Nigeria.
\end{abstract}

Key words: ships of death; waste trafficking; environmental crime; ecomafie; Italian-Nigerian diplomatic relations.

L'articolo affronta il tema del traffico internazionale di rifiuti tossico-nocivi italiani diretto in Nigeria tra il 1987 e il 1988, alla luce dei rapporti diplomatici intrattenuti dai governi italiani con il Paese destinatario durante la seconda metà degli anni Ottanta. Il contributo pone al centro dell'analisi la rotta italo-nigeriana - quella maggiormente utilizzata dalle società che organizzarono ed effettuarono i traffici durante gli anni Ottanta . gli attori che ne presero parte, la merce che fu scaricata e le importanti, e finora poco studiate, ricadute sul piano dei rapporti bilaterali tra Italia e Nigeria.

Parole chiave: navi dei veleni; traffico di rifiuti; criminalità ambientale; ecomafie; rapporti diplomatici italo-nigeriani. 


\section{Introduzione}

Tra il 1987 e il 1988 nove imbarcazioni cariche di rifiuti tossici partirono dai porti italiani facendo rotta verso Paesi del Sud del Mondo con l'intento di smaltire in loco gli scarti prodotti dalle industre italiane. Da quanto emerge dalla documentazione approvata e pubblicata dalla Commissione parlamentare d'inchiesta sul ciclo dei rifiuti (da ora in avanti solo Commissione rifiuti), della diciassettesima legislatura, che al tema ha dedicato una relazione apposita, ${ }^{1}$ ben cinque spedizioni ebbero una comune destinazione: il porto fluviale di Koko, in Nigeria. Proprio nel Paese governato da Ibrahim Babangida, però, le cose non andarono come previsto. Quando nel giugno del 1988 le autorità nigeriane scoprirono l'esistenza di questi traffici e l'utilizzo di terreni agricoli al posto di discariche, richiesero immediatamente il recupero dei fusti al governo italiano creando tensioni diplomatiche particolarmente rilevanti. ${ }^{2}$

Questo saggio intende portare l'attenzione sulla rotta nigeriana del traffico internazionale di rifiuti tossici-nocivi, la più inflazionata delle cosiddette "navi dei veleni", sugli attori che organizzarono ed effettuarono i traffici tra il 1987 e il 1988, e sulle importanti criticità diplomatiche che emersero nell'estate del 1988 tra il governo nigeriano di Ibrahim Babangida e il governo italiano presieduto da Ciriaco De Mita. ${ }^{3}$

\footnotetext{
${ }^{1}$ Si veda: Commissione parlamentare d'inchiesta sul ciclo dei rifiuti, XVII legislatura, Relazione sulle «navi dei veleni». I traffici internazionali di rifiuti negli anni ' 80 e '90, doc. XXIII N. 51, approvata il 28 febbraio 2018.

${ }^{2}$ Si vedano: Sylvia F. Liu, The Koko Incident: Developing International Norms for the Transboundary Movement of Hazardous Waste, in "Journal of Natural Resources \& Environmental Law", vol. 8, no. 1, 1992-1993, pp. 121-154; Robert Lu, West Africa; The Industrial World's Dumping Grounds, in "Harvard International Review", vol. 11, n. 4, Summer 1989, pp. 57-59; Phil 0'Keefe, Toxic Terrorism, in "Review of African Political Economy", n. 42 (1988), pp. 84-90; Cyril I. Obi, Globalization and Environmental Conflict in Africa, in "African Journal of Political Science / Revue Africaine de Science Politique", Vol. 4, No. 1 (June 1999), pp. 40-62; Toyin Falola, Adam Paddock, Environment and Economics in Nigeria, Abingdon, Routledge, 2013.

${ }^{3}$ All'interno di questo saggio viene proposta una parte dei risultati della ricerca dottorale condotta tra il 2017 e il 2020 che ha portato alla stesura della tesi dal titolo: Ships of death. Il traffico internazionale di rifiuti tossico-nocivi e radioattivi italiani diretto in Libano, Nigeria e Somalia (19871992).
} 


\section{La ricerca}

Il tema ha ricevuto particolare attenzione da parte degli studiosi intorno ai primi anni Novanta per poi esser affrontato a partire dai primi anni del nuovo millennio. ${ }^{4}$ È possibile individuare ricerche provenienti da molteplici e differenti campi di studi oltre a una importante mole di dossier di associazioni ambientaliste e inchieste giornalistiche. ${ }^{5}$ In questo dialogo a più voci, contributi di rilievo sono stati forniti seguendo approcci differenti: dagli studi giuridici alle scienze politiche e sociali, passando per gli studi sulla criminalità organizzata, fino agli studi storici. ${ }^{6}$ Nell'ultimo decennio un riferimento a parte lo merita la green criminology nel suo importante tentativo di far convergere l'attenzione di studiosi afferenti da aree diverse delle scienze politiche, sociali e storiche attorno allo studio del fenomeno della criminalità ambientale. ${ }^{7}$

Più complicato, invece, è rinvenire una produzione scientifica di tipo storico per ciò che concerne i traffici marittimi di rifiuti. ${ }^{8}$ Considerazioni di più ampio respiro si possono rintracciare all'interno degli studi sulla globalizzazione e sulle politiche intraprese dai Paesi industrializzati nei confronti dei Paesi del Sud del Mondo.

\footnotetext{
${ }^{4}$ Significative ricerche furono stilate nei primi anni Novanta. Tra gli altri, si vedano: Mostafa K. Tolba, Osama El-Kholy, The World Environment 1972-1992. Two decades of challenge, Oxford, Springer Science\&Business Media, 1992; Christoph Hilz, The International Toxic Waste Trade, New York, Van Nostrand Reinhold, 1992.

5 Solo per citare alcuni importanti dossier: Greenpeace, Waste Trade in The Mediterranean - Toxic Attack Against Lebanon Case One: Toxics from Italy, August 1996; Greenpeace, The Toxic Ships. The Italian hub, the Mediterranean area and Africa, 2010; Legambiente, Le nuove frontiere dell'ecomafia (1994-1997), 29 gennaio 1997.

${ }^{6}$ In merito allo scenario globale si veda: Bill Moyers, Global Dumping Ground. The International Traffic in Hazardous Waste, Cambridge, The Lutterworth Press, 1991; Muawya Ahmed Hussein, Costs of environmental degradation: An analysis in the Middle East and North Africa region, in "Management of Environmental Quality. An International Journal", vol. 9, n. 3, anno 2008, pp. 305-317; M. K. Tolba, O. El-Kholy, Op. cit;; Christoph Hilz, The International Toxic Waste Trade, New York, Van Nostrand Reinhold, 1992; Kofi D. Asante-Duah, Imre V. Nagy, International Trade in Hazardous Waste, New York, E\&FN Spon, 1998; Jan Albers, Responsibility and Liability in the Context of Transboundary Movements of Hazardous Wastes by Sea, London, Springer, 2015.

${ }^{7}$ In merito alla green criminology, tra i tanti, si vedano: Tanya Wyatt, (a cura di) Hazardous Waste and Pollution. Detecting and Preventing Green Crimes, London, Springer, 2016; Alan A. Block, Environmental Crime and Pollution: Wasteful Reflections, in "Social Justice", Vol. 29, No.1/2, (87-88), Globalization and Environmental Harm (2002), pp. 61-81; Rob White, Transnational Environmental Crime. Toward an eco-global criminology, Abingdon, Routledge, 2011.

${ }^{8}$ Si veda: John R. McNeill, Qualcosa di nuovo sotto il sole. Storia dell'ambiente nel XX secolo, Torino, Einaudi, 2002; Gabriella Corona, Breve storia dell'ambiente in Italia, Il Mulino, Bologna, 2015. Tra i contributi storici che affrontano il problema ambientale, si menziona anche: Ugo Biliardo, Giuseppe Mureddu, Maree nere: rischio ambientale, in Il mosaico Mediterraneo, Carlo M. Santoro (a cura di), Bologna, Il Mulino, Bologna, 1991, pp. 261-323; Cecilia Mastrantonio, Il punto di vista ambientalista sulla proposta di Craxi sul debito dei Paesi poveri, in Ambiente Italia 1991, Giovanna Melandri, Giulio Conte (a cura di), Mondadori, Milano, 1991, p.201 e ss.
} 
All'interno di questa letteratura, infatti, si è posta particolare attenzione anche ai danni ambientali causati o incentivati da politiche di cooperazione progettate e attuate non sempre in piena armonia con il territorio e con le richieste della popolazione locale. ${ }^{9}$ Particolarmente circoscritta, però, sembra essere l'attenzione verso una ricostruzione storica del fenomeno in questione, nonostante il tema si presti, a tutti gli effetti, ad un'analisi di questo tipo. Sono evidenti i danni ambientali e sanitari causati dallo stoccaggio o dallo smaltimento di rifiuti pericolosi nei luoghi in cui questi furono introdotti illegalmente, così come lo sono le connessioni con lo sviluppo industriale dei maggiori produttori di scarti tossici, tutelati spesso da normative nazionali troppo morbide e da pene irrisorie o, talvolta, solo pecuniarie, per i trasgressori. ${ }^{10}$ Ciò che, però, nel corso degli anni è passato inosservato è la valenza politica e diplomatica assunta dai traffici in questione.

Nel tentativo di inserirsi in quest'ambito cercando di apportare qualche elemento innovativo di analisi, si intende fornire uno studio della rotta maggiormente utilizzata dalle società di import-export italiane durante la seconda metà degli anni Ottanta e delle conseguenze diplomatiche che ne seguirono nell'estate del 1988. Quali, quindi, gli attori che organizzarono ed effettuarono i traffici lungo la rotta nigeriana? E quali le criticità diplomatiche emerse nell'estate del 1988 tra i Paesi protagonisti del traffico?

Per rispondere agli interrogativi di ricerca sono state consultate una serie di fonti, edite e inedite, che è opportuno descrivere. Fonti edite significative ai fini di questa ricerca sono state: le Relazioni delle Commissioni rifiuti, ${ }^{11}$ i Testi e documenti sulla

\footnotetext{
${ }^{9}$ Si vedano, in particolare, gli studi di Sara Lorenzini e il lavoro di ricognizione sulla Cooperazione allo Sviluppo formulato da Valerio Bini: Sara Lorenzini, Una strana guerra fredda. Lo sviluppo e le relazioni Nord-Sud, Il Mulino, Bologna, 2017, p. 204 e ss.; Sara Lorenzini, Global Development. A Cold War History, Princeton University Press, Princeton, 2019; Valerio Bini, La cooperazione allo sviluppo in Africa. Teorie, politiche, pratiche, Mimesis, Milano-Udine, 2016, p. 18 e ss.

10 Solo per citarne alcuni: Jennifer Clapp, The Toxic Waste Trade with Less-Industrialised Countries: Economic Linkages and Political Alliances. Third World Quarterly, vol. 15, no. 3, 1994, pp. 505-518; Muawya Ahmed Hussein, Costs of environmental degradation: An analysis in the Middle East and North Africa region, cit.; Rob White, Toxic Cities: Globalizing the Problem of Waste, in "Social Justice", Vol. 35, No. 3 (113), War, Crisis \& Transition (2008-09), pp. 107-119.

${ }^{11}$ In particolare, il riferimento va a tre Relazioni della Commissione: Relazione sulla morte del capitano di fregata Natale De Grazia, doc. XXIII n.18, approvata il 5 febbraio 2013 (XVI legislatura); Relazione sul fenomeno delle "navi a perdere», doc. XXIII n.21, approvata il 28 febbraio 2013 (XVI legislatura); Relazione sulle «navi dei veleni». I traffici internazionali di rifiuti negli anni ' 80 e '90, cit. (XVII legislatura).
} 
politica estera dell'Italia pubblicati dall'ex Ufficio Studi del Ministero degli Affari Esteri e della Cooperazione Internazionale e, ancora, i dossier di Associazioni ambientaliste italiane e internazionali, i report redatti dall'United Nations Environment Programme. Accanto a esse, i rapporti presentati da gruppi e partiti politici, consultati a Roma presso l'Archivio Centrale dello Stato (da ora in avanti ACS) e presso l'Archivio Storico della Camera dei Deputati (da ora in avanti ASCD).

Per quel che concerne le fonti primarie inedite sia di tipo diplomatico che giudiziario, sono stati utilizzati: telegrammi e appunti presenti nel Fondo della Presidenza del Consiglio dei Ministri, Ufficio del Consigliere Diplomatico (II Parte) e relazioni e dossier versati da Partiti e Gruppi parlamentari italiani e conservati all'interno dei rispettivi fondi - il riferimento va, in particolare, ai fondi del Partito della Rifondazione Comunista e del Gruppo Parlamentare Sinistra Indipendente, tutti conservati presso l'ASC -. Per quel che concerne l'ASCD, l'attenzione è stata rivolta soprattutto alla documentazione versata dalle Commissioni parlamentari d'inchiesta sul ciclo dei rifiuti. Relativamente agli Archivi di natura giudiziaria, le principali fonti sono state consultate presso le Procure di Paola e di Asti. All'interno dell'Archivio della Procura calabrese, ho rintracciato una scambio di telegrammi tra la Farnesina e l'Ambasciatore italiano in Libano Antonio Mancini avvenuto nell'estate del 1988. Si è trattato di un ritrovamento che mi ha permesso di andare più in profondità nella vicenda del ritrovamento dei rifiuti italiani in Libano, rinvenuti contemporaneamente rispetto a quelli individuati in Nigeria, e mi ha consentito di raccogliere informazioni utili per comprendere quel processo decisionale che portò gli organi competenti a effettuare il recupero dei fusti in entrambi i Paesi.

Presso l'Archivio della Procura di Asti sono stati consultati i faldoni afferenti al procedimento penale n.395/97, al cui interno è stata ritrovata documentazione particolarmente preziosa per ricostruire il processo di ingresso e stoccaggio dei rifiuti in Nigeria e le società che operarono in loco.

L'articolo si struttura in modo tale da inserire la rotta italo-nigeriana nel quadro della movimentazione globale dei rifiuti tossici -secondo paragrafo - e all'interno dei rapporti di politica estera tra l'Italia e la Nigeria - terzo paragrafo - ponendo 
l'attenzione su una prima e spesso trascurata fase di criticità diplomatiche tra i due Stati che precede di soltanto un anno la crisi dell'88. È questo lo scenario dentro cui si sviluppano le vicende analizzate nei successivi paragrafi dove si riportano i risultati della ricerca relativamente alla rotta italo-nigeriana, agli attori e alle spedizioni che l'hanno composta, e alle criticità diplomatiche successive alla scoperta dei fusti contenenti rifiuti tossico-nocivi stoccati sul suolo nigeriano.

\section{Alle origini del traffico marittimo di rifiuti: le "navi dei veleni"}

Prima di percorrere la direttrice italiana del traffico internazionale di rifiuti tossici durante la seconda metà degli anni Ottanta, occorre fare un riferimento a quanto accadde nel decennio precedente, collocandoci a cavallo tra la seconda metà degli anni Settanta e i primi anni Ottanta, quando vennero alla luce i primi casi di movimentazione internazionale di rifiuti pericolosi. ${ }^{12}$ Proprio in quegli anni, infatti, si instaurò un "potente equilibrio economico" tra la richiesta di abbattimento dei costi di smaltimento, proveniente dai produttori dei rifiuti, e l'offerta di smaltimento in aree periferiche del Paese o, peggio ancora, del mondo.

Grazie a importanti studi pubblicati soprattutto negli anni Novanta e, fino ad oggi, ritenuti punti di riferimento per gli studiosi nel settore, è possibile individuare una commistione di fattori che, da un lato, stimolò le industrie a ricercare soluzioni di smaltimento particolarmente vantaggiose, seppur illegali, e, dall'altro, incentivò le proposte di smaltimento all'estero di intermediari, società di import-export e colletti bianchi. ${ }^{13}$ Facendo uno sforzo di sintesi, è possibile individuare e schematizzare i seguenti fattori:

\footnotetext{
${ }^{12} \mathrm{Si}$ veda il caso dei fratelli Colbert in: Simone M. Müller, Hidden Externalities: The Globalization of Hazardous Waste, in "Business History Review", n. 93 (spring 2019), pp. 51-74; B. Moyers, op. cit. 13 Monica Massari, Paola Monzini, Dirty Businesses in Italy: A Case-study of Illegal Trafficking in Hazardous Waste, in "Global Crime", Vol. 6 n. 3-4, August-November 2004, pp.285-304, p. 286; K. D. Asante-Duah, F. F. Saccomanno, J. H. Shortreed, The Hazardous Waste Trade: can It be controlled?, cit., p. 1685; J. Clapp, Toxic Exports, cit.; C. Hilz, op. cit;; Lori Gilmore, The Export of non-hazardous waste in "Environmental Law", vol. 19, No. 4 (Summer 1989), pp. 879-907.
}

Cross Vol.7 N² (2021) - DOI: http://dx.doi.org/10.54103/cross-16848 


\section{La ricerca}

I. Aumento della produzione: nella prima metà degli anni Ottanta, la produzione di rifiuti pericolosi nei paesi industrializzati aumentò esponenzialmente rispetto a quella del decennio precedente - un valore che oscilla tra il 1020\% e il 2800\%; ${ }^{14}$

II. Regolamentazione del ciclo dei rifiuti e definizioni: tra la fine degli anni Settanta e i primi anni Ottanta, i Paesi industrializzati si dotarono di norme che regolamentavano lo smaltimento, classificavano i rifiuti e, conseguentemente, gli impianti di trattamento e/o i siti di smaltimento. In Italia solo il 10 settembre del 1982, in risposta alle direttive europee della seconda metà degli anni Settanta, ${ }^{15}$ si attuò il primo storico tentativo di classificazione dei rifiuti - ad eccezione di quelli radioattivi - suddividendoli in urbani, speciali e tossiconocivi. ${ }^{16}$

III. Riduzione spazi di smaltimento: con l'entrata in vigore di queste prime norme nazionali e con il sovrapporsi dell'aumento di produzione di rifiuti pericolosi, si riducono gli spazi (in valore assoluto) di trattamento o smaltimento sul territorio di produzione.

Questi fattori causarono un aumento vertiginoso dei prezzi di smaltimento dei rifiuti - costi totalmente a carico del produttore - all'interno dei Paesi industrializzati. Tra la fine degli anni Settanta e i primi anni Ottanta, il costo di smaltimento nei paesi produttori di rifiuti si attestava tra i $100 \$$ per tonnellata, con un massimo di $250 \$$ per lo smaltimento in discarica e intorno ai 500\$ per l'incenerimento - prezzi che, per giunta, aumentarono nella seconda metà degli anni Ottanta. ${ }^{17}$ Mancando una definizione e una regolamentazione comune e globale di cosa fossero e come

\footnotetext{
${ }^{14}$ Il primo dato proviene da: K. D. Asante-Duah, I. V. Nagy, International Trade in Hazardous Waste, cit., p.70. Per quanto concerne il secondo dato, si vedano: M. K. Tolba, O. El-Kholy, op. cit., p. 264; United Nations, General Assembly, 44th session, 18 July 1989 (A/44/362), Development and International Economic Co-Operation: Environment. Illegal traffic in toxic and dangerous products and wastes, p.6.

15 Il riferimento va alle direttive: n. 75/442/Cee del 15 luglio 1975; n. 76/403/Cee del 6 aprile 1976; n. 78/319/Cee del 20 marzo 1978.

${ }^{16}$ Riflessioni critiche sulle direttive Cee e sul loro recepimento in Italia sono presenti in: Nicola Sarti, Normativa comunitaria ed internazionale sui rifiuti con particolare riguardo alla prevenzione ed al movimento transfrontaliero dei rifiuti, in Rischio rifiuti. Atti del convegno nazionale del 23 gennaio 1988 a Legnano, Amedeo Amadei, Marino Bottini, Gabriella Pecchenini, (a cura di), Centro Stampa Olgiati, Legnano, 1989, pp. 105-108. Prima del Dpr 915/82, vi fu un tentativo di regolamentazione degli scarichi in mare con la legge n. 319 del 10 maggio 1976, cosiddetta Legge Merli, in reazione, anche in questo caso, alle indagini e alle prime sentenze del Tribunale di Livorno in merito al caso dei fanghi di Scarlino. Sul punto si veda: Tullio Scovazzi, Immersione di sostanze inquinanti in mare e risarcimento del danno, in "Rivista giuridica dell'ambiente", 1986, fascicolo 1, pp. 99-108.

${ }^{17}$ Si vedano: K. D. Asante-Duah, F. F. Saccomanno, J. H. Shortreed, The Hazardous Waste Trade: can It be controlled?, cit., p. 1685; C. Hilz, op. cit., p. 46.
} 
andassero trattati gli hazardous waste, smaltire la medesima quantità e tipologia di rifiuti nella gran parte dei Paesi in via di sviluppo garantiva un notevole abbattimento dei costi. Si consideri, infatti, che il costo di smaltimento in Guinea come in Libano, in Nigeria come in Venezuela, variava tra i 2,5\$ e i $40-50 \$$ per tonnellata. ${ }^{18}$

Dati i fattori menzionati, la movimentazione marittima intercontinentale dei rifiuti di produzione industriale si palesò quale soluzione vantaggiosa per i produttori dei rifiuti e remunerante e priva di rischi penali per gli attori che se ne occuparono.

In questo scenario globale è possibile inserire il contesto italiano e, in particolare, la vicenda delle "navi dei veleni". Rispetto all'arco di tempo indicato nel precedente paragrafo, in Italia sembrerebbe che le conseguenze dell'intreccio tra i fattori poc'anzi menzionati emersero a distanza di qualche anno. Solo a partire dai decreti attuativi del 1985, il d.p.r. 915/82, l'unica norma nazionale istituita per categorizzare i rifiuti e definirne i criteri di classificazione, divenne pienamente operativa. ${ }^{19}$ La Commissione rifiuti della diciassettesima legislatura, a tal proposito, sottolineò che "il decreto crea, nello stesso tempo, una cultura giuridica e d'indagine rispetto al tema" sicché, essendoci a quel punto una normativa di riferimento, "alcune autorità giudiziarie e polizie specializzate (...) iniziarono a monitorare il settore". ${ }^{20}$ Solo a partire dalla seconda metà degli anni Ottanta, il fenomeno venne alla luce in modo sempre più evidente ma ciò non può indurre ad escludere che, prima di allora, non vi fossero stati casi di traffici transfrontalieri di rifiuti tossici via mare. Proprio in quegli anni, però, le discariche e gli impianti di trattamento italiani versassero in condizioni critiche.

Stando a quanto evidenziato dalla Commissione rifiuti, le strutture italiane riuscivano ad accogliere regolarmente circa il 10\% dei rifiuti tossici prodotti

\footnotetext{
${ }^{18}$ C. Hilz, op. cit., p. 46; Royal Institute of International Affairs, Sustainable Development Programme, International Environmental Crime. The Nature and Control of Environmental Black Markets, Background paper for RIIA workshop, 27-28 May 2002, p.17; Donald Liddick, Crimes Against Nature. Illegal Industries and the Global Environment, Praeger, Santa Barbara, 2011, pp.13-40, p.14.

${ }^{19}$ Commissione parlamentare d'inchiesta sul ciclo dei rifiuti, XVII legislatura, Relazione sulle «navi dei veleni», cit., p. 10.

${ }^{20}$ Ibidem.
} 
annualmente dal comparto nazionale. ${ }^{21}$ Problema che si amplificò durante la seconda metà degli anni Ottanta. Dalla ricerca condotta è emerso, infatti, che in Italia la produzione di rifiuti pericolosi continuò ad aumentare di circa il 90\% rispetto al quinquennio precedente. ${ }^{22}$

È in questo quadro, dunque, che si inserì la movimentazione transfrontaliera di rifiuti tossici quale una delle offerte di mercato che parte del comparto industriale italiano colse per ridurre i costi di smaltimento degli scarti prodotti.

Soffermandoci solo sulle spedizioni transfrontaliere via mare di cui si ha contezza, la Commissione rifiuti quantificò un totale di sei imbarcazioni organizzate da un'unica società e partite esclusivamente da porti toscani verso Libano, Nigeria e Venezuela, tra il febbraio del 1987 e aprile-maggio del 1988.23 Il comun denominatore di queste spedizioni era rappresentato dalla divisione ecologica della Jelly Wax S.r.l di Opera, produttrice di paraffine. Oltre a queste è possibile aggiungere almeno altre tre spedizioni effettuate dalla "concorrente" Sirteco S.r.l. e dalla Piattaforma Ecologica Industriale.

Tabella 1 - Navi dei veleni 1987-1988

\begin{tabular}{|l|l|l|l|}
\hline Nave & Partenza & Destinazione & Data partenza \\
\hline Lynx & M. di Carrara & $\begin{array}{l}\text { Puerto Cabello } \\
\text { (Venezuela) }\end{array}$ & Febbraio 1987 \\
\hline Akbay 1 & M. di Carrara & Sulina (Romania) & Aprile 1987 \\
\hline Corina & Chioggia & Sulina (Romania) & Aprile 1987 \\
\hline Radhost & M. di Carrara & Beirut (Libano) & Giugno 1987 \\
\hline Baru Luck & M. di Carrara & Koko (Nigeria) & Luglio-agosto 1987 \\
\hline Danix & Darsena di Pisa & Koko (Nigeria) & Settembre 1987 \\
\hline
\end{tabular}

21 Ivi, p.47.

22 World Resources Institute, World Resources, 1990-91, Basic Books, New York, 1991; Organisation for Economic Co-operation and Development, Environmental Indicators, OECD, Paris, 1991.

${ }^{23}$ Commissione parlamentare d'inchiesta sul ciclo dei rifiuti, XVII legislatura, Relazione sulle «navi dei veleni», cit., pp. 17-18.

Cross Vol.7 N² (2021) - DOI: http://dx.doi.org/10.54103/cross-16848 
La ricerca

\begin{tabular}{|l|l|l|l|}
\hline Line & Darsena di Pisa & Koko (Nigeria) & Novembre-dicembre 198724 \\
\hline Line & Darsena di Pisa & Koko (Nigeria) & Marzo 1988 \\
\hline Jorgen Vesta & Livorno & Koko (Nigeria) & Aprile-maggio 1988 \\
\hline
\end{tabular}

La tabella n.1 consente di mettere in risalto diversi elementi utili per l'analisi. Il porto di Marina di Carrara sembra esser stato la punta di diamante per le esportazioni della Jelly Wax e delle altre ditte a essa collegate. La Lynx prima, la Radhost e la Baru Luck poi, partirono tra il febbraio ed il luglio dell'87, inizialmente con la medesima destinazione, Puerto Cabello. ${ }^{25}$ Includendo la Akbay 1 si ha un totale di quattro imbarcazioni dirette in quattro paesi diversi: Venezuela, Romania, Libano e, in ultimo, Nigeria.

Peraltro, dal medesimo porto, partirono navi con carichi non correttamente dichiarati nelle bolle, senza mai arrivare nei porti di destinazione, le quali si presume trasportassero scarti radioattivi. È il caso, ad esempio, della Michigan partita nell'ottobre del 1986 da Marina di Carrara e affondata a largo della costa tirrenica calabrese o, ancora, della Rigel, partita dal medesimo porto il 9 settembre 1987 e affondata il 21 settembre 1987 a largo di Capo Spartivento. ${ }^{26}$

Per quel che concerne i Paesi destinatari, dalla tabella emerge un netto predominio della rotta nigeriana rispetto alle altre. Più della metà delle spedizioni individuate avevano come destinazione la Nigeria. Pretesto per lo studio di questa rotta, però, non si spiega solo con la quantità di "navi dei veleni" di cui fu destinazione. A ciò, infatti, si aggiunge un elemento di tipo storico-politico.

Quando, nel giugno del 1988, le autorità nigeriane si resero conto di quel che stava accadendo, grazie alle denunce di giornalisti e cittadini, il governo presieduto da Ibrahim Babangida si mosse per attuare delle contromisure tali da incentivare l'Italia a recuperare i fusti tossici precedentemente inviati. Nell'estate del 1988 le

\footnotetext{
${ }^{24}$ La partenza della prima spedizione effettuata con la Line, secondo la Relazione sulle «navi dei veleni», andrebbe datato ai primi di dicembre.

${ }^{25}$ Commissione parlamentare d'inchiesta sul ciclo dei rifiuti, XVII legislatura, Relazione sulle «navi dei veleni», cit., p. 30.

${ }^{26}$ Commissione parlamentare d'inchiesta sul ciclo dei rifiuti, XVI legislatura, Relazione sul fenomeno delle «navi a perdere», doc. XXIII n.21, approvata il 28 febbraio 2013, p.22.
}

Cross Vol.7 №2 (2021) - DOI: http://dx.doi.org/10.54103/cross-16848 
tensioni diplomatiche tra il governo nigeriano e quello italiano, allora presieduto da Ciriaco De Mita, sfociarono nel richiamo in patria dell'Ambasciatore nigeriano in Italia, James Tsado Kolo, e nel sequestro di un'imbarcazione battente bandiera italiana, la Piave, in sosta nel porto di Lagos. Ma in quale scenario si inseriscono queste azioni diplomatiche? Attraverso il paragrafo successivo si tenta di delineare il quadro dei rapporti tra i due Paesi prima della crisi del 1988, portando l'attenzione su una prima e spesso trascurata fase di tensioni tra i due governi, ricostruita all'interno della ricerca. ${ }^{27}$

\section{Uno sguardo ai rapporti diplomatici tra Italia e Nigeria negli anni}

\section{Ottanta}

Dalla ampia letteratura visionata, dalla consultazione de L'Italia nella politica internazionale prodotto annualmente dall'Istituto Affari Internazionali e dai Testi $e$ documenti sulla politica estera dell'Italia, preziosa fonte edita dal Servizio Storico e Documentazione del Ministro degli Affari Esteri, ${ }^{28}$ per quel che concerne i primi anni Ottanta, emergono dei rapporti diplomatici improntati soprattutto sulla politica commerciale e di cooperazione allo sviluppo. Da quando, nel 1979, la Nigeria aveva superato la Libia per quantità di petrolio prodotto, divenendo il maggior produttore in Africa, e il settimo al mondo, ${ }^{29}$ l'Italia aveva rafforzato i rapporti con il paese. Un "mini-boom petrolifero" che incentivò un repentino decollo dell'industrializzazione, generando forti divergenze di reddito e portando lo Stato nigeriano a contrarre accordi per la costruzione di opere di dubbia utilità. 30

\footnotetext{
27 Il riferimento va, in particolare, al Capitolo 4 della tesi di dottorato, intitolato: "Navi dei veleni: rifiuti per Koko e Beirut".

${ }^{28}$ Consultabile presso: http://www.farnesina.ipzs.it/biblioteca/testiDocumenti/4.

${ }^{29}$ Istituto Affari Internazionali, L'Italia nella politica internazionale 1979-1980, Roma, Edizioni di Comunità,1981, p. 271.

30 Istituto Affari Internazionali, L'Italia nella politica internazionale 1983-1984, Franco Angeli, Milano, 1986, p.509; Marcella Emiliani, Petrolio, forze armate e democrazia. Il caso Nigeria, Carocci, Roma, 2004, p.116; René Dumont, Démocratie pour l'Afrique, (1 ed. 1991), trad. It. Democrazia per l'Africa, Elèuthera, Milano, 1992, p.169. In merito al debito nigeriano, si vedano le tabelle della World Bank: World Debt Tables: external debt of developing countries (1991-1992), Washington, 1991.
} 
In questo scenario si inserisce, tra il 1986 e il 1987, una prima fase di tensioni diplomatiche tra i governi italiano e nigeriano, trascurata persino dalla Commissione rifiuti, ${ }^{31}$ che meriterebbe particolare attenzione data l'affinità temporale con i traffici oggetto d'analisi.

Il debito pubblico nigeriano aumentava di oltre il 30\% e il governo nigeriano fu costretto a sospendere di un anno i pagamenti per le aziende terze. Tra queste, alcune società italiane con le quali aveva accumulato un debito totale di oltre trentasei miliardi di lire. Entro i primi di ottobre del 1986 sarebbe dovuta partire una prima tranche di pagamenti, ma le cose andarono diversamente, poiché allo scadere della data ultima nessun risarcimento fu evaso e il governo nigeriano dovette dichiarare la mancanza di divisa, bloccando il trasferimento di denaro dalla Central Bank of Nigeria. ${ }^{32}$ A seguito dell'intermediazione della Farnesina, la filiale di Londra della Chase Manhattan Bank, - per conto della banca nigeriana - avviò le procedure di pagamento emettendo delle promissory notes. ${ }^{33}$ Si trattava, però, di promesse di pagamento inesigibili in Italia.

Il 3 dicembre del 1986 Peter Onu, Direttore generale del Ministero degli Esteri nigeriano - e, all'epoca, Presidente della delegazione per le consultazioni politiche italo-nigeriane - incontrò a Roma il Sottosegretario agli Affari Esteri Mario Raffaelli. Nonostante le criticità, dal comunicato della Farnesina non emerse nulla di rilevante, e Onu espresse pieno soddisfacimento per i rapporti diplomatici tra i due Paesi. ${ }^{34} \mathrm{Al}$ contempo, però, alcune società italiane creditrici avviarono azioni legali.

\footnotetext{
31 Il riferimento è alla Relazione sulle «navi dei veleni» della Commissione rifiuti della XVII legislatura, più volte citata nelle precedenti pagine.

32 Senato della Repubblica, X Legislatura, Disegno di legge n. 969 d'iniziativa dei senatori Azzaretti, Angeloni e Meriggi (DC-PCI) comunicato alla presidenza il 7 aprile 1988 sulle "Misure per garantire il pagamento dei crediti vantati da imprese italiane nei confronti di clienti nigeriani". https://www.senato.it/service/PDF/PDFServer/DF/267934.pdf. Da questo documento si legge che le ditte maggiormente esposte - con dati in miliardi di lire - erano la Ilsa International S.r.l. di Milano (10,8 mld), la Elba \& C. S.p.A. di Vigevano (4,8 mld), la Iemmepi S.r.l. di Milano (3,2 mld) e a seguire due ditte massesi, la Teico S.p.A. (3 mld) e la Carrara Export G.O.C. S.r.l. (2,9 mld). Poco esposta era la Finesport Engineering S.r.l. di Roma (0,68 mld), azienda socio dell'Istituto per Cooperazione Economica Internazionale e i Problemi dello Sviluppo.

33 Ibidem.

${ }^{34}$ Ministero degli Affari Esteri, Testi e documenti sulla politica estera italiana - 1986, Roma, Istituto Poligrafico e Zecca dello Stato, 1990, p. 232
} 
Il 28 luglio del 1987, con l'intento di incentivare la riscossione del denaro di cui le aziende erano creditrici, l'autorità giudiziaria sostenne la richiesta effettuata da società creditrici, ed effettuò un sequestro conservativo della River Kerawa, nave cargo battente bandiera nigeriana, appartenente alla Nigerian National Shipping Line e ancorata nel porto di La Spezia. ${ }^{35}$ Passarono pochi giorni e le autorità nigeriane sequestrarono manu militari due imbarcazioni italiane nel porto di Lagos, la Cielo di Napoli e la Jolly Nero, con l'accusa di "aver violato le leggi nigeriane entrando nelle acque territoriali senza che le autorizzazioni di sicurezza necessarie fossero state concesse." 36 L'intervento del Ministero di Giustizia presieduto da Giuliano Vassalli e, in seguito, della Farnesina - con l'impegno di farsi carico del problema dei crediti italiani con il Governo nigeriano - raffreddarono gli animi, e le tre navi sequestrate, con i rispettivi equipaggi, poterono riprendere il largo. ${ }^{37}$

È questo, dunque, lo scenario in cui occorre inserire la rotta nigeriana, che prese avvio proprio in quell'estate del 1987 con l'approdo della prima imbarcazione, la Baru Luck, nel porto fluviale di Koko, ed è in questo clima politico "polemico", 38 tra il regime di Babangida ed i governi Fanfani e Goria, che occorre inserire le tensioni diplomatiche del 1988 tra l'Italia e la Nigeria.

\footnotetext{
${ }^{35}$ Istituto Affari Internazionali, L'Italia nella politica internazionale 1987-1988, Franco Angeli, Milano, 1990, p. 570. Il sequestro dell'imbarcazione fu avallato dal Tribunale di Pisa quale "unico bene nigeriano al momento presente entro i confini italiani". Si veda: Senato della Repubblica, XIII Legislatura, Disegno di legge n. 4499 d'iniziativa dei senatori Servello, Basini, Magliocchetti, Turini, Demasi e Pontone comunicato alla presidenza il 24 febbraio 2000 http://www.senato.it/japp/bgt/showdoc/13/Ddlpres/0/00005757/index.html?part=doc_dc. 36 Istituto Affari Internazionali, L'Italia nella politica internazionale 1987-1988, cit., p. 570.

37 Si veda: Senato della Repubblica, XIII legislatura, $163^{\circ}$ seduta (pomeridiana), 3 aprile 1997, Resoconto Stenografico, Interrogazione parlamentare (n. 4-05147) dei senatori Montagna e Marchetti al Presidente del Consiglio dei ministri e ai Ministri degli affari esteri e per gli italiani all'estero e di grazia e giustizia, pp. 96-99.

${ }^{38}$ Istituto Affari Internazionali, L'Italia nella politica internazionale 1987-1988, cit., p. 570.
} 


\section{La rotta nigeriana}

Dalla consultazione delle fonti inedite conservate presso l'ASCD, e versate dalla Commissione rifiuti, è emerso che la rotta nigeriana era composta da cinque spedizioni partite da tre porti italiani tra il 1987 e il 1988, con un carico totale di poco inferiore alle quattromila tonnellate di rifiuti tossici. ${ }^{39}$ Dati, questi, che ridimensionano notevolmente il quantitativo ipotizzato dalla Commissione rifiuti della diciassettesima legislatura e, prima ancora, da Greenpeace nel report dal titolo Toxic Ships, che ipotizza un carico complessivo di 43.330 tonnellate diviso in cinque spedizioni effettuate da quattro navi. ${ }^{40}$ Dati differenti vengono consegnati dalle Nazioni Unite che segnalano la partenza di cinque navi con rifiuti tossici e radioattivi per un totale di 18.000 fusti nel 1987.41

Tabella 2 - La rotta nigeriana (1987-1988)

\begin{tabular}{|l|l|l|l|}
\hline Nave & Partenza & Arrivo iniziale & Arrivo finale \\
\hline Baru Luck & M. di Carrara & Puerto Cabello & Koko \\
\hline Danix & Darsena di Pisa & Koko & Koko \\
\hline Line & Darsena di Pisa & Sulina & Koko \\
\hline Line & Darsena di Pisa & Koko & Koko \\
\hline Jorgen Vesta & Livorno & Koko & Koko \\
\hline
\end{tabular}

Oltre a una differenza di dati, le fonti consultate presso l'ASCD consentono di mettere in rilievo un ulteriore elemento: la merce caricata sulla Baru Luck e sulla prima spedizione della Line non sarebbe dovuta giungere in Nigeria bensì in Venezuela e Romania. A imporre un cambio di rotta furono le inchieste e le attenzioni mediatiche che emersero attorno ai casi venezuelano e rumeno.

\footnotetext{
${ }^{39}$ Medesimi dati sono presenti in: S. F. Liu, The Koko Incident, cit., p.131.

${ }^{40}$ Commissione parlamentare d'inchiesta sul ciclo dei rifiuti, XVII legislatura, Relazione sulle «navi dei veleni», cit., p.18; Greenpeace, The Toxic Ships, cit.

41 United Nations, General Assembly 44th session, 18 July 1989 (A/44/362), Development and International Economic Co-Operation, cit. p.22; United Nations, Security Council, 66 ${ }^{\text {th }}$ session, 25 October 2011, Report of the Secretary-General on the protection of Somali natural resources and waters, p. 12.
} 
Tra le due spedizioni, però, c'è una differenza sostanziale: la prima era stata organizzata dalla Jelly Wax - con la complicità di società italo-elvetiche - mentre la seconda era stata pianificata dalla Sirteco S.r.l., la "concorrenza" della Jelly. Questa società, infatti, era solita organizzare le proprie esportazioni lungo la rotta rumena che, però, tra l'aprile e il maggio del 1987, si sarebbe interrotta. È quanto sostenne in un'intervista Claudio Bertacin, amministratore della Sirteco S.r.l., il quale disse inoltre che, dopo aver capito che "in Romania qualcosa non funzionava", si decise a "dirottare la spedizione sulla Nigeria", accordandosi con i suoi "concorrenti" che gestivano la rotta nigeriana. ${ }^{42}$ Proprio per tale ragione, i rifiuti presenti sulla Line, nave battente bandiera della Germania Occidentale, partirono poi per Koko e furono "sepolti da qualche parte" in Nigeria. ${ }^{43}$

Bertacin e la Sirteco dovettero quindi accordarsi con la società cardine dei traffici diretti in Nigeria, la Jelly Wax S.r.l., all'epoca amministrata da Renato Pent. La divisione ecologica della società, intorno alla metà degli anni Ottanta, era attiva nell'esportazione, stoccaggio e smaltimento di rifiuti industriali-farmaceutici italiani ed effettuò diverse spedizioni transfrontaliere di rifiuti.

Renato Pent, ascoltato dalla Commissione rifiuti della sedicesima legislatura, riferì di esser stato il primo "a esportare rifiuti con le navi", vantando il rapporto fiduciario instauratosi tra le industrie e la Jelly Wax basato, a suo dire, sul fatto che la società lavorasse "correttamente". ${ }^{44}$ Cosa intendeva, con ciò, l'amministratore della Jelly Wax?

È verosimile ritenere che ciò su cui si basava questo rapporto fiduciario fosse il rilascio della documentazione attestante l'avvenuto smaltimento dei fusti. Attraverso l'utilizzo di documentazione apposita si riusciva "a certificare quell'apparenza solo cartacea di corretta gestione dei rifiuti inviati." ${ }^{45}$ La presenza

\footnotetext{
${ }^{42}$ Claudio Bertacin, Vita e miracoli del "re delle navi pattumiera", intervista di Claudio Sabelli Fioretti per "Il Secolo XIX" del 13 settembre 1988, documento 26, fascicolo 4, Commissione parlamentare d'inchiesta sul ciclo dei rifiuti e sulle attività illecite ad esso connesse XIII legislatura, ASCD.

43 Ibidem.

${ }^{44}$ Commissione parlamentare d'inchiesta sul ciclo dei rifiuti della XVI legislatura, Audizione di Renato Pent, Bologna, 17 febbraio 2010, p.2.

${ }^{45}$ Commissione parlamentare d'inchiesta sul ciclo dei rifiuti, XVII legislatura, Relazione sulle «navi dei veleni». I traffici internazionali di rifiuti negli anni ' 80 e '90, doc. XXIII N. 51, approvata il 28 febbraio 2018, p. 91.
} 
di documenti formalmente validi attestanti, almeno sulla carta, la liceità della movimentazione e dello smaltimento, fungeva da garanzia, per il produttore dei rifiuti, del corretto smaltimento. Poco importava se la documentazione rilasciata attestasse uno smaltimento che, in realtà, non era avvenuto. Contava la documentazione.

In Nigeria, la società destinataria dei fusti era la Iruekpen Construction Company (da ora in avanti, ICC) con sede a Lagos, il cui Consigliere delegato era un impresario edile toscano, Gianfranco Raffaelli. Costui, oltre a esser destinatario dei rifiuti tramite la ICC, collaborava con la Jelly Wax nella fase di esportazione o intermediazione marittima attraverso una seconda società, la S. I. Ecomar S.r.l. di Novi Ligure, amministrata insieme a un avvocato. In sostanza, l'invio di rifiuti italiani in Nigeria effettuato dalla Jelly Wax con la collaborazione o l'intermediazione della S. I. Ecomar, poteva esser facilmente eluso avendo come impresa importatrice la ICC.

Il rapporto di fiducia tra le industrie e la Jelly Wax si basava, quindi, sia sulla documentazione fornita alle industrie, sia sull'apparente legittimità del percorso che effettuavano i rifiuti, alla luce dei contratti stipulati con imprese del paese destinatario che si sarebbero dovute occupare dello smaltimento. A tal proposito, questa ricerca consente di aggiungere un ulteriore tassello.

All'interno della documentazione consultata presso la Procura di Asti, è stato possibile rinvenire un permesso di importazione datato 18 maggio 1987, rilasciato dall'Office of the Pharmacists Board of Nigeria che consentiva alla ICC di importare "industrial and laboratory chemicals" in Nigeria e affidava alla società la possibilità "to import and clear" detta merce, in una o più soluzioni, nell'arco dell'anno solare. ${ }^{46}$ Si noti, inoltre, un ulteriore dettaglio all'interno del suddetto permesso. Il verbo utilizzato all'interno della concessione, "to clear", lascia abbondantemente aperta la possibilità dello smaltimento di una tipologia di rifiuti che, all'interno dello stato nigeriano, non era possibile smaltire, data la mancanza di strutture idonee. Il

\footnotetext{
${ }^{46}$ Office of the Pharmacists Board of Nigeria, Autorizzazione di importazione n. 676, per Iruekpen Construction Company Nig. Ltd. di Gianfranco Raffaelli, 18 maggio 1987, fascicolo del procedimento penale n. 747/96 (afferente al 395/97), Archivio della Procura di Asti (da ora in avanti, APAs). Il documento è stato richiamato anche in: P. O'Keefe, Toxic Terrorism, cit., p. 86.
} 
documento precisa, inoltre, che la società di Raffaelli avrebbe potuto beneficiare dell'ausilio della Triana Ltd. - segnalata, evidentemente, dallo stesso Raffaelli - per quel che concerneva la fase di movimentazione.

È ragionevole supporre, dunque, che proprio attraverso questo permesso, la Jelly Wax e la Ecomar riuscirono a oltrepassare i limiti normativi, classificando i rifiuti in maniera difforme rispetto a ciò che in realtà erano, così da poterli introdurre sul suolo nigeriano usufruendo del permesso poc'anzi citato.

Nel medesimo faldone in cui è stato rinvenuto il permesso d'importazione, è stato individuato un secondo documento di particolare rilievo. Si tratta della dichiarazione di Raffaelli, presso l'Alta Corte di Giustizia di Lagos, di aver importato e smaltito sostanze chimiche in Nigeria, grazie al permesso regolarmente rilasciato dalle autorità nigeriane. Dal documento, datato 12 ottobre 1987, emerge che Raffaelli importò dalla S.I. Ecomar di Novi Ligure "residual products of the chemical or allied industries" e che, prosegue, "I have dumped in to my premises situated in Bendel State of Nigeria". ${ }^{47}$ Raffaelli aveva scaricato rifiuti chimici all'interno di una sua proprietà collocata nell'ex Stato di Bendel. In questo Stato, riferisce Raffaelli, si trovava il luogo in cui aveva scaricato i rifiuti industriali sdoganati nel porto di Koko. ${ }^{48}$ Non si trattava, però, della presunta sede della ICC di Asaba. ${ }^{49}$

Giunti al porto di Koko e scaricati dalle navi, una parte dei fusti fu stoccata sul terreno agricolo di un abitante del luogo, Sunday Nana. Sull'identità di questo soggetto non si hanno molte certezze: potrebbe essersi trattato di un semplice contadino o taglialegna o, come segnalato dal giornalista Andrea Palladino, di un uomo d'affari. ${ }^{50}$ Non vi sono dubbi, invece, sull'accordo stipulato da Raffaelli con Nana nel quale si prevedeva il deposito di gran parte dei fusti sul terreno di

\footnotetext{
47 Gianfranco Raffaelli, Dichiarazione presso l'Alta Corte di Giustizia di Lagos, 12 ottobre 1987, fascicolo del procedimento penale n. 747/96 (afferente al 395/97), APAs.

48 Office of the Pharmacists Board of Nigeria, Autorizzazione di importazione n. 676, per Iruekpen Construction Company Nig. Ltd. di Gianfranco Raffaelli, 18 maggio 1987, cit.

49 Ibidem.

50 Per quanto concerne la prima ipotesi si veda: S. F. Liu, The Koko Incident: Developing International Norms for the Transboundary Movement of Hazardous Waste, cit., p. 131. Per la seconda si veda: Andrea Palladino, Bandiera nera. Le navi dei veleni, Manifestolibri, Roma, 2010, p. 71. Circa Sunday Nana e la vicenda di Koko si segnalano anche: C. Hilz, op. cit., p.29; Greenpeace, Toxic Ships, cit., p. 11; R. Lu, West Africa; The Industrial World's Dumping Grounds, cit., p. 57.
} 
proprietà del cittadino nigeriano per soli $100 \$$ al mese. Un comune terreno agricolo trasformato in discarica di rifiuti pericolosi di oltre una trentina di aziende italiane. ${ }^{51}$

\subsection{Le spedizioni}

Tra l'estate del 1987 e la primavera del 1988, dai porti toscani partirono cinque spedizioni di rifiuti tossico-nocivi prodotti da industrie italiane. Ben poco è possibile dire in merito alla prima nave che solcò le acque nigeriane, la Baru Luck. È documentato che la nave imbarcò la merce nel porto di Marina di Carrara, con l'intenzione di movimentarla fino alle coste venezuelane, e scaricarla a Puerto Cabello. Dopo la crisi ambientale scoppiata in loco a seguito dello scarico dei rifiuti della nave Lynx (tab.1), la società di esportazione optò per un cambio di destinazione poco prima della partenza della nave.

Un quadro più completo e dettagliato è delineabile in merito alle spedizioni partite dalla darsena di Pisa, attraverso il prezioso dossier redatto da Democrazia Proletaria dal titolo "Pisa-Koko: il traffico dei rifiuti in Nigeria", e consegnato dall'On. Edo Ronchi durante la seduta della Camera dei Deputati del 14 luglio 1988.52

La prima delle spedizioni pisane fu effettuata con la Danix, un'imbarcazione battente bandiera danese, diretta a Koko. Dal dossier è emerso che l'Agenzia Marittima Bonistalli di Livorno, in ventiquattr'ore, ricevette il nullaosta per l'imbarco merci firmato dal sindaco del Comune di Pisa, autorità competente per la darsena. Tra il 2 e il 5 settembre, sulla Danix furono imbarcate poco meno di duecento tonnellate di rifiuti tossico-nocivi. ${ }^{53}$ Dal dossier trapelano, inoltre, i nomi delle industrie proprietarie dei rifiuti e delle aziende intermediarie, tra cui ne emerge una di

\footnotetext{
${ }^{51}$ S. F. Liu, The Koko Incident, cit, p. 134. Si veda anche: José Ramos Regidor, Pagar es morir, vogliamo vivere, in "Nigrizia", gennaio 1989, F. 1042 "Amazzonia", Busta 107, Fondo del Partito della Rifondazione Comunista, ACS.

${ }^{52}$ Democrazia Proletaria, Dossier "Pisa-Koko: il traffico dei rifiuti in Nigeria", consegnato dall'On. Edo Ronchi durante la seduta del 14 luglio 1988, Camera dei Deputati, X legislatura.

53 Giacomino Granchi, Nullaosta per Agenzia Marittima Bonistalli, 2 settembre 1987, Allegato1, Democrazia Proletaria, Dossier "Pisa-Koko: il traffico dei rifiuti in Nigeria", 14 luglio 1988, cit., p. 17082.
} 


\section{La ricerca}

particolare rilievo: la Ecomovil di Pianfei. Dalla documentazione consultata presso l'ASCD e presso la Procura di Paola, risulta che solo pochi anni dopo l'esportazione a Koko, la Ecomovil effettuò trasporti di rifiuti pericolosi tramite la Tanagro Trasporti di Luigi Cardiello, pluripregiudicato e trasportatore di rifiuti, anche su commissione di gruppi camorristici, in discariche abusive campane e lucane. ${ }^{54}$

Il primo dicembre del 1987, una seconda imbarcazione partì da Pisa. 876 tonnellate di rifiuti tossici, nocivi e speciali furono caricate tra il 14 ed il 16 novembre a bordo della Line, battente bandiera della Germania Occidentale, con destinazione iniziale il porto franco di Sulina - modificata durante la navigazione verso Koko. Anche in questo caso fu l'Agenzia Marittima Bonistalli a richiedere al comune di Pisa il nullaosta, ricevendolo in meno di ventiquattr' ore. ${ }^{55}$ Scarti di lavorazione di pesticidi, resine, morchie di vernici e vari altri rifiuti industriali, furono movimentati da ditte di trasporti con licenze scadute o sprovvisti di specifica autorizzazione e, in alcuni casi, diffidati dalle rispettive Regioni per il trasporto di rifiuti pericolosi. ${ }^{56}$

Intorno ai primi giorni del nuovo anno, precisamente il 9 gennaio, anche la rotta nigeriana rischiò di interrompersi. L'Amministrazione Provinciale di Pisa notò delle anomalie nella movimentazione della merce dalla darsena e inviò al Sindaco di Pisa un rapporto redatto dai funzionari dell'Ufficio Ambiente in cui si legge che

"i sopralluoghi e le verifiche effettuate da questo Ufficio e la documentazione qui pervenuta, su richiesta, dalle Regioni di Provenienza del rifiuto (Piemonte, Lombardia e Veneto) hanno evidenziato un consistente traffico di rifiuti tossici e nocivi di varia natura e provenienza, verso il Porto Darsena in attesa di imbarco con destinazione paesi extracomunitari quali Romania e Nigeria." ${ }^{57}$

\footnotetext{
${ }^{54}$ Prefettura di Matera, Relazione in merito allo smaltimento dei rifiuti pericolosi nel materano, 13 novembre 1995, documento 1, Fascicolo 80, Commissione Parlamentare d'inchiesta sul ciclo dei rifiuti e sulle attività illecite ad esso connesse, XII legislatura, ASCD.

55 Giacomino Granchi, Nullaosta per Agenzia Marittima Bonistalli, 14 novembre 1987, Allegato 2, Democrazia Proletaria, Dossier di "Pisa-Koko: il traffico dei rifiuti in Nigeria", 14 luglio 1988, cit., p. 17085.

56 Democrazia Proletaria, Dossier di "Pisa-Koko: il traffico dei rifiuti in Nigeria", 14 luglio 1988, cit., pp. 17068-17069.

57 Ufficio Ambiente, Amministrazione Provinciale Pisa, Rapporto diretto ad Assessore Regionale, Dipartimento Uso ed Assetto del Territorio Regione Toscana ed al Sindaco del Comune di Pisa, 9 gennaio 1988, Allegato 4, Democrazia Proletaria, Dossier di "Pisa-Koko: il traffico dei rifiuti in Nigeria", 14 luglio 1988, cit., p. 17092.
} 
Il 5 marzo 1988, come se nulla fosse, un nuovo nullaosta fu firmato dalle autorità pisane per l'imbarco di altri rifiuti tossico-nocivi sulla Line, con destinazione Koko. A bordo della nave battente bandiera della Repubblica Federale di Germania, secondo quanto emerge dal dossier redatto da Democrazia Proletaria, vi erano carichi provenienti da tre esportatori diversi: 844 t. esportate dalla Sirteco; $771 \mathrm{t}$. dalla Ecomar e 117 t. da un'altra società, la Ekoground. Il carico della Sirteco sarebbe dovuto partire con la Kaptan Fehmi da Marina di Carrara nel giugno 1987 ma fu bloccato dal Pretore per manifeste irregolarità. Il tutto fu poi inviato a Pisa e caricato il 16 marzo sulla Line. ${ }^{58}$ Per quanto concerne il carico della Ecomar di Raffaelli, è interessante notare come, tra le ditte che effettuarono questa spedizione, vi fossero sia industrie sia altre società di intermediazione sprovviste di autorizzazioni o con diffide regionali. Tra queste vi era la Ecomovil di Pianfei, intermediaria per un carico di circa trecento tonnellate, che il 23 dicembre 1987 ricevette una diffida dalla Regione Piemonte. ${ }^{59}$

Oltre alla ditta cuneese, Raffaelli esportò anche rifiuti presi in carico dalla Ecolife S.a.s di Milano, una società che svolgeva attività di intermediazione collocandosi, in sostanza, tra il produttore del rifiuto e l'esportatore. ${ }^{60}$ La Ecolife, come la Sirteco e la Jelly Wax, raccolse rifiuti pericolosi provenienti da grandi industrie italiane, esportando sia in Nigeria che in Libano. ${ }^{61}$ Anche in quel caso, le ditte che effettuarono i trasporti verso la Darsena di Pisa risultarono essere, in gran parte, senza regolare autorizzazione. ${ }^{62}$

Sul finire di aprile, da Livorno, partì una quinta nave. Dalla documentazione primaria consultata sono poche le informazioni che emergono con chiarezza in merito a questo carico. ${ }^{63}$ La P.E.I. risultò essere esportatrice di circa 700 t. caricate

\footnotetext{
58 Ivi, p. 17075; Claudio Bertacin, "Vita e miracoli del "re delle navi pattumiera"”, cit.

${ }^{59}$ Democrazia Proletaria, Dossier di "Pisa-Koko: il traffico dei rifiuti in Nigeria", 14 luglio 1988, cit., p. 17077.

60 Ibidem.

61 Ibidem. Si vedano: Karim S. Makdisi, Implementing International Environmental Treaties in Developing Countries: Using Flows to Explain How Lebanon Dealt with the Trade in Hazardous Waste, Paper Presented at the International Studies Association Annual Meeting 2006, San Diego, California, 23 March 2006, p. 5; A. Palladino, Bandiera nera, cit., p. 71.

62 Democrazia Proletaria, Dossier di "Pisa-Koko: il traffico dei rifiuti in Nigeria", 14 luglio 1988, cit.

63 Si veda: Democrazia Proletaria, Dossier di "Pisa-Koko: il traffico dei rifiuti in Nigeria", 14 luglio 1988, cit.; Gruppo Consiliare di Democrazia Proletaria della Regione Liguria, Dossier "Rumenta Story.
}

Cross Vol.7 N² (2021) - DOI: http://dx.doi.org/10.54103/cross-16848 
sulla Jorgen Vesta, l'ultima "nave dei veleni" diretta a Koko. Nonostante le attenzioni di una parte delle autorità politiche fin dal novembre 1987, la Jorgen Vesta riuscì a partire senza troppe difficoltà.

\subsection{Gli attori}

Dallo studio effettuato, emerge un quadro ben definito in merito agli attori che interagirono e ai meccanismi che si attivarono per effettuare i traffici. La Jelly Wax e le altre società esportatrici organizzarono e gestirono la rotta nigeriana potendo fruire di importanti e, a volte, poco chiari legami professionali e istituzionali. A effettuare il servizio di export furono divisioni ecologiche di aziende o società di intermediazione o, ancora, proprio società di import-export. Costoro furono il terminale delle industrie, da un lato, e, tramite le società di brokeraggio marittimo, di tutto ciò che concerneva la spedizione via mare, l'esportazione, lo smaltimento in Nigeria e il rilascio della documentazione certificante l'avvenuto smaltimento. Per quel che concerneva il trasporto della merce sul territorio italiano è emerso che, quasi fosse la prassi, le industrie utilizzarono trasportatori privi di regolare autorizzazione $\mathrm{o}$, al più, scaduta. ${ }^{64}$

Il rapporto tra industria e trasportatori, all'interno dei casi nigeriani, non sembrerebbe essere stato mediato da broker o da società di intermediazione. Era l'industria, dunque, a mantenere i contatti con i trasportatori. Il consistente utilizzo di ditte di trasporti mancanti di adeguate autorizzazioni al trasporto dei rifiuti non può che far sorgere almeno qualche dubbio sulla malafede nella movimentazione degli scarti da parte delle industrie interessate.

Grazie al dossier di Democrazia Proletaria è stato possibile mettere in luce come i rifiuti, una volta giunti all'interno dell'area portuale della darsena, furono fatti entrare senza alcun preavviso e senza controlli ma con regolare autorizzazione al

Il traffico dei rifiuti dall'Italia all'estero", settembre 1988, documento 12, Fascicolo 4, Commissione parlamentare d'inchiesta sul ciclo dei rifiuti e sulle attività illecite ad esso connesse, XIII legislatura, ASCD.

${ }^{64}$ Democrazia Proletaria, Dossier "Pisa-Koko: il traffico dei rifiuti in Nigeria”, cit., p. 17069 ss. 
deposito e all'imbarco. I fusti furono momentaneamente immagazzinati in un'area concessa all'Agenzia Marittima Bonistalli, per poi esser caricati su una nave individuata, presumibilmente, dalla S.I. Ecomar. ${ }^{65}$ Era questa, infatti, la società che si occupava dell'intermediazione marittima.66 Attori integranti e componenti la società di intermediazione erano, come detto, gli avvocati, che potrebbero aver stretto rapporti con le Compagnie di navigazione scelte per i traffici e che furono coinvolti - nel caso della S.I. Ecomar - o complici, nell'esportazione.

Dalla documentazione rinvenuta presso la Procura di Asti è emerso che Raffaelli, oltre ad aver svolto il ruolo di intermediario, fu, almeno sulla carta, il destinatario dei rifiuti sul territorio nigeriano. All'interno dei documenti di esportazione, come visto nelle pagine precedenti, figurava il nome della ICC di cui Raffaelli era Consigliere Delegato. In Nigeria, però, non furono introdotti "industrial and laboratory chemicals"67 bensì rifiuti tossici industriali italiani.

Meno di due settimane dopo l'arrivo al porto di Koko della Jorgen Vesta, negli stessi giorni in cui nel Parlamento italiano si discuteva il cosiddetto Decreto Enichem, in Nigeria scoppiava il "Koko case". ${ }^{6}$

\section{Dentro una crisi diplomatica}

Dal 2 giugno del 1988, in Nigeria, le testate giornalistiche locali e nazionali prestarono particolare attenzione al traffico dei rifiuti pericolosi partito dall'Italia dando risalto mediatico ad affari illeciti che avrebbero coinvolto anche funzionari nigeriani. Il 5 giugno, il quotidiano nigeriano The Guardian pubblicò un articolo dal

\footnotetext{
${ }^{65}$ Ivi, p. 17072.

66 Gruppo Consiliare di Democrazia Proletaria della Regione Liguria, Dossier "Rumenta Story. Il traffico dei rifiuti dall'Italia all'estero", settembre 1988, cit.

67 Office of the Pharmacists Board of Nigeria, Autorizzazione di importazione n. 676, per Iruekpen Construction Company Nig. Ltd. di Gianfranco Raffaelli, 18 maggio 1987, cit.

${ }^{68}$ L'Enichem era uno dei tre presidi industriali - insieme alla Montedison di Porto Marghera e alla Sibib di Scarlino - a cui era consentito lo scarico diretto in mare. Si veda: Giovanna Melandri (a cura di), Ambiente Italia. Rapporto 1989: dati, tendenze, proposte, Isedi Petrini Editore, Torino, 1989.
} 
titolo "Toxic Waste Dump in Koko Town", in cui forniva ulteriori informazioni in merito a Sunday Nana e Raffaelli. 69

La reazione del governo nigeriano fu immediata e, tra il 5 e il 7 giugno, si sviluppò su diversi fronti. Sul versante internazionale, il governo comunicò all'Organisation of African Unity (da ora, Oau) e all'Unep il traffico e lo smaltimento di rifiuti che vedeva interessate le terre nigeriane. Al contempo, il Ministro degli Affari Esteri Ike Nwachukwu richiese l'intervento del governo italiano per il recupero dei rifiuti, giudicando "poco amichevole" il comportamento assunto dall'Italia. ${ }^{70}$ Sul fronte interno, venne approvato il decreto 42/88 istituendo la Federal Environmental Protection Agency che avrebbe dovuto dettare le linee guida su cosa fosse o meno un rifiuto pericoloso e su come andasse smaltito. ${ }^{71}$

Il 10 giugno, di fronte alle indecisioni del Parlamento italiano e del Ministro dell'Ambiente Giorgio Ruffolo, il governo nigeriano impose il sequestro della motonave Piave, battente bandiera italiana, di proprietà della compagnia di navigazione Lloyd Triestino, in sosta nel porto di Lagos. ${ }^{72}$ Dapprima le autorità nigeriane intendevano utilizzare l'imbarcazione italiana per effettuare il recupero dei fusti scaricati a Koko, ma si trattava di una portacontainer di circa centonovanta metri di lunghezza e, con ogni probabilità, sarebbe bastato percorrere le prime miglia del Benin River per rendere inevitabile un disastro navale. Stando a quanto

\footnotetext{
${ }^{69}$ Oluwafemi A. Ladapo, The Contribution of Cartoonists to Environmental Debates in Nigeria: The Koko Toxic-Waste-Dumping Incident, in "RCC Perspectives", n. 1, Eco-Images: Historical Views and Political Strategies, 2013, pp.61-72, p. 61.

${ }^{70}$ Istituto Affari Internazionali, L'Italia nella politica internazionale 1987-1988, cit., p. 571; S. F. Liu, The Koko Incident: Developing International Norms for the Transboundary Movement of Hazardous Waste, cit., p. 131.

${ }^{71}$ Questa specifica azione normativa intrapresa dal governo fu un atto meramente formale perché il Fepa divenne operativo solo con il decreto 59 del 1992. Si veda: Edward-Ekpu Douglas Uwagbale, Hazardous Waste Management and Challenges in Nigeria, in "Public Health International", 1 (1), 2016, pp. 1-5.

${ }_{72}$ Si veda: Antonio Cianciullo, Enrico Fontana, Dark Economy. La mafia dei veleni, Einaudi, Torino, 2012, p.129; Istituto Affari Internazionali, L'Italia nella politica internazionale 1988-1989, Franco Angeli, Milano, 1990, p. 571; R. Lu, West Africa; The Industrial World's Dumping Grounds, cit., p. 57; A. Palladino, Bandiera Nera, cit., p. 65; Greenpeace, The Toxic Ships cit., p. 10; C. Hilz, op. cit., p. 31. Secondo alcuni studiosi, oltre alla Piave, fu bloccata anche la Danix che in passato trasportò proprio i rifiuti diretti a Koko e in quegli stessi giorni di giugno si trovava nel porto di Lagos. Alla Danix fu immediatamente consentito di ripartire per via del fatto che era una nave battente bandiera danese. Si veda: S. F. Liu, The Koko Incident: Developing International Norms for the Transboundary Movement of Hazardous Waste, cit., p. 131.
} 
riferito a Radio Radicale da Giorgio Bascherini, secondo di coperta della Piave, nonostante il clima sulla nave fosse particolarmente teso, i marinai italiani e il loro comandante riuscirono a far capire alle autorità nigeriane il problema tecnico che impediva loro di arrivare fino a Koko per recuperare i fusti. ${ }^{73}$ Il disastro fu evitato ma la nave e l'equipaggio rimasero sotto sequestro per oltre un mese. ${ }^{74}$

Nel frattempo, fu avviata un'indagine che in pochi giorni portò all'arresto di cittadini nigeriani - tra le venti e le quaranta persone - e di Desiderio Perazzi, braccio destro di Gianfranco Raffaelli. ${ }^{75}$ Nel medesimo giorno del sequestro della Piave, il governo nigeriano richiamò in patria l'ambasciatore a Roma James Tsado Kolo, "for immediate consultations". ${ }^{76} \mathrm{Si}$ era aperta una vera e propria crisi diplomatica tra l'Italia e la Nigeria. Non c'è dubbio che l'operazione di recupero dei fusti, richiesta a gran voce dalle autorità nigeriane, fosse quella auspicata, ma è chiaro che implicava un esborso economico elevato, sia per quel che concerneva il recupero e il rientro dei rifiuti, sia per il loro smaltimento. Si trattava, in sostanza, di individuare una o più società che avrebbero noleggiato una o più navi su cui sarebbero stati caricati $\mathrm{i}$ fusti, per poi essere trasportati in Italia, previa individuazione di un porto apposito e, cosa principale, andavano individuate o edificate strutture atte a poterne smaltire il contenuto tossico. Nel frattempo, però, i ventiquattro marinai italiani a bordo della Piave erano sotto sequestro nelle acque nigeriane.

Solo intorno alla metà di luglio il Ministro Lattanzio e i rappresentati del Ministero degli esteri e dell'Ambiente assegnarono ad Ambiente S.p.A. - società del gruppo Eni - la gestione del noleggio nave e del recupero dei fusti a Koko. Il compito di EniAmbiente era quello di recuperare i fusti, riportarli in Italia, bonificare l'area e

\footnotetext{
${ }^{73}$ Intervista realizzata da Bruno Luverà per Radio Radicale a Giorgio Bascherini, marinaio della Piave il 2 agosto 1988. L'intervista è consultabile su: https://www.radioradicale.it/scheda/28253/scorieradioattive-italiane-in-nigeria-liberata-la-nave-piave-bloccata-per-ritorsione.

74 Ibidem.

${ }^{75}$ Si veda: L. Gilmore, op. cit., p. 884.

${ }^{76}$ S. F. Liu, The Koko Incident: Developing International Norms for the Transboundary Movement of Hazardous Waste, cit., p.131. Si veda anche: Istituto Affari Internazionali, L'Italia nella politica internazionale 1987-1988, cit., p.571; R. Lu, West Africa; The Industrial World's Dumping Grounds, cit., pp. 57-59; C. Hilz, op. cit., p. 31.
} 


\section{La ricerca}

procedere al conferimento e allo smaltimento dei rifiuti. Facendo ricorso ai fondi della Cooperazione allo Sviluppo, fu possibile avviare le pratiche. ${ }^{77}$

Il 18 luglio, Eni-Ambiente noleggiò la Karin B e, a seguire, la Deepsea Carrier e la Hai Xiong. ${ }^{78}$ Le prime due navi si sarebbero dovute occupare del recupero dei fusti mentre la terza avrebbe dovuto trasportare in Italia il terreno contaminato decorticato. Nella sostanza, era facile ipotizzare che, una volta recuperati i fusti e bonificate le aree in cui questi erano stati stoccati, la Piave e il suo equipaggio avrebbero potuto riprendere il largo. Di fatto, solo il 25 luglio 1988 la Piave poté levare l'ancora e rientrare in Italia. Pochi giorni dopo, anche la Karin $B$, carica dei fusti trasportati dalla società italiane, poté fare rotta verso l'Italia.

Risolte le criticità sulla sponda nigeriana, era necessario risolvere quelle in Italia poiché nessun porto voleva accogliere le "navi dei veleni" di rientro. Dal 29 maggio, il porto di Genova, sotto il controllo della Castalia e del Commissario ad acta Giuseppe Francese, ospitava la Zanoobia con i fusti spediti in Venezuela dalla Jelly Wax e da altre società. Con l'ingresso della Karin B e della Deepsea Carrier in acque italiane ai primi di settembre, la protesta divampò a Livorno e Ravenna, i porti in cui sarebbero dovute approdare le due navi. Ad aggravare il tutto, la difficoltà di rinvenire dei siti di smaltimento idonei per il materiale rientrante da Koko. Come se non bastasse, l'Italia aspettava il rientro di due navi dal Libano con i fusti scaricati a Beirut. $^{79}$

In una settimana, tra il 9 ed il 16 settembre, il Governo De Mita aprì il programma di emergenza per la gestione dei rifiuti di rientro, varando, nell'immediato, due decreti-legge. Il 9 settembre fu la volta del decreto-legge n. 397 con “Disposizioni

\footnotetext{
77 Ministero degli Affari Esteri, Appunto indirizzato all'Ufficio XIV della Direzione Generale per la Cooperazione allo Sviluppo e per conoscenza all'Ufficio VI della Dgcs ed all'Ufficio IX della Direzione Generale degli Affari Economici, 18 luglio 1988, Allegato 31, Informativa del 21 ottobre 1997 della Polizia Giudiziaria di Reggio Calabria, Sottofascicolo 36, Faldone 3, APPa.

${ }^{78}$ Dossier a cura del Centro Informativo "Karin B", dicembre 1989, Documento 2, Fascicolo 52, Commissione parlamentare d'inchiesta sul ciclo dei rifiuti e sulle attività illecite ad esso connesse XIII legislatura, ASCD.

${ }^{79}$ Ordinanza del 21 settembre 1988 del Ministro per il Coordinamento della Protezione Civile Vito Lattanzio, (Ordinanza n. 1561/FPC), "Ulteriori disposizioni eccezionali afferenti alle operazioni finalizzate allo smaltimento definitivo delle sostanze tossiche e nocive trasportate dalla nave Karin B". (GU Serie Generale n.231 del 01-10-1988).
} 
urgenti in materia di smaltimento dei rifiuti industriali", seguito dall'avvio del programma di emergenza e dall'entrata in vigore, il 16 settembre, del decreto del Presidente del Consiglio dei Ministri per l'“'Individuazione dei siti e delle modalità per lo stoccaggio e lo smaltimento controllato dei rifiuti industriali tossici trasportati da navi". ${ }^{80}$ Era stata accertata "una situazione di emergenza nascente dalla necessità di far approdare urgentemente in porto italiano le navi Karin B e Deep Sea Carrier, nonché una terza nave proveniente dalla Nigeria" che "per la loro tossicità richiedono lo smaltimento urgente". ${ }^{81}$

A metà settembre la Karin B e la Deepsea Carrier giunsero in acque territoriali italiane, e avviarono le operazioni di sbarco dell'equipaggio e di scarico della nave nel porto di Livorno. Per gestire queste operazioni, il Ministro Lattanzio e il Ministro Ruffolo designarono, quali Commissari ad acta per la gestione della vicenda nigeriana, il Presidente del Consiglio Regionale dell'Emilia-Romagna Luciano Guerzoni e il suo omologo della Regione Toscana Gianfranco Bartolini. Alla task force da loro istituita e coordinata, il compito di gestire il rientro, lo scarico, il trasporto nei siti di stoccaggio e, se fosse stato necessario, anche la costruzione di idonei siti di smaltimento. Nuove discariche furono create e un intero piazzale di stoccaggio nel porto di Ravenna fu completato e collaudato. ${ }^{82}$

Con il recupero dei fusti, le tensioni diplomatiche con il governo nigeriano si avviarono verso una risoluzione nell'arco di pochi mesi, con il rientro a Roma dell'ambasciatore nigeriano James Tsado Kolo sul finire di agosto. Occorrerà aspettare i primi anni Novanta per una distensione completa e definitiva.

\footnotetext{
${ }^{80}$ I riferimenti in Gazzetta Ufficiale sono: GU Serie Generale n. 213 del 10-09-1988; GU Serie Generale n. 218 del 16-09-1988.

${ }^{81}$ Si veda: Ordinanza del 21 settembre 1988 del Ministro per il Coordinamento della Protezione Civile Vito Lattanzio, (Ordinanza n. 1561/FPC), cit.

82 Per i dettagli tecnici, si veda: Demetrio Egidi, Stefano Vannini, Le navi dei veleni e le bonifiche dei siti contaminati. Dalla gestione dell'emergenza una metodologia di intervento, Pitagora Editrice S.r.l., Bologna, 1995, pp. 187-229.
} 


\section{La ricerca}

\section{Conclusioni}

Attraverso lo studio qui presentato si è cercato di far emergere l'importanza della rotta nigeriana sia sotto il profilo dello studio storico dei traffici di rifiuti italiani, sia per quello politico-diplomatico. Le fonti primarie hanno portato alla luce i meccanismi dei traffici diretti in Nigeria, consentendo di esaminare approfonditamente le tre spedizioni di rifiuti tossici che partirono dalla Darsena di Pisa. Merce che ricevette regolare autorizzazione all'imbarco dalle autorità pisane.

È stato possibile fare luce sugli attori che effettuarono i traffici da cui è emerso un "ventaglio ampio di relazioni"83 tra poteri pubblici locali, operatori economici, professionisti di vario tipo, unitamente al complesso mondo dell'industria. All'interno delle società che si occuparono dell'import-export sia sul versante italiano che su quello nigeriano è emerso come avvocati e broker finanziari fornirono il loro know how alle società in questione e ad altri attori presenti nella rete.

Per quel che concerne gli attori, un interrogativo rimane aperto. La politica - italiana e nigeriana - ebbe un ruolo all'interno di questi meccanismi? Perché le autorità nigeriane che autorizzarono la ICC all'importazione della merce non effettuarono regolari controlli nei confronti della società in questione? Si trattò di "semplice" negligenza da parte delle autorità nigeriane? Si tratta di domande che andrebbero poste alle autorità italiane che diedero il nulla osta per il carico dei fusti, consapevoli che si trattasse di merce di scarto industriale.

I componenti di Democrazia Proletaria, all'interno del dossier Pisa-Koko, sottolinearono più volte come il Sindaco pisano avesse autorizzato l'imbarco dei fusti in tempi record rispetto alla norma - in sole ventiquattr'ore - inducendo a ritenere che vi fosse una complicità da parte del Sindaco o quanto meno, a chiedersi

\footnotetext{
${ }^{83}$ Gabriella Corona, Rocco Sciarrone, Il paesaggio delle ecocamorre, in "Meridiana. Rivista di storia e scienze sociali", No. 43, Reti di mafie (2002), pp.13-35, p. 22. Per la precisione, Corona e Sciarrone fanno riferimento alle reti di relazioni attraverso le quali le organizzazioni criminali condizionano le scelte urbanistiche e i piani regolatori ma è un concetto applicabile senza alcuna forzatura, a mio avviso, anche nel campo dei traffici dei rifiuti.
} 
se fu la fretta a non permettere a costui di effettuare i dovuti controlli. ${ }^{84}$ In tal senso, la negligenza, da un lato, del Sindaco pisano nel firmare quelle autorizzazioni all'imbarco della merce e, dall'altro, delle autorità nigeriane nel rilasciare alla ICC il permesso di importazione di quel tipo di merce, forse, senza effettuare i dovuti controlli, ha senza dubbio facilitato il compito delle società che organizzarono il traffico, effettuarono l'esportazione della merce in Nigeria e la stoccarono, e in parte la smaltirono, in loco.

Le autorità italiane competenti nella fase di controllo dell'esportazione dei rifiuti, da quel 9 gennaio del 1988, non poterono più dire di non sapere: ciò che si stava svolgendo - senza se e senza ma - era un traffico internazionale di rifiuti pericolosi con annessa autorizzazione e nullaosta delle autorità italiane. Le diffide inviate tra il novembre e il dicembre del 1987 alle ditte di trasporto dei rifiuti ed alle aziende intermediarie, documentano la consapevolezza e il tentativo, meramente formale, di parte delle istituzioni italiane di negare l'autorizzazione a questo traffico. ${ }^{85}$ Le pagine di questo dossier denunciarono come le istituzioni italiane avrebbero potuto fare di più nel tentativo di bloccare questo traffico in essere. La domanda, allo stato attuale senza risposta, è se vi furono delle responsabilità o, peggio, delle complicità da parte delle autorità politiche italiane che oliarono la macchina burocratica rendendo possibili questi traffici.

In conclusione: questo articolo ha consentito di mettere in luce i meccanismi e gli attori che si attivarono lungo la rotta maggiormente utilizzata all'interno del traffico internazionale di rifiuti tossici che ha avuto luogo durante la seconda metà degli anni Ottanta. Lo studio del traffico in oggetto, inserito in uno scenario di più ampio respiro, ha consentito di delineare la genesi della movimentazione globale dei rifiuti tossici lungo la rotta Nord-Sud del mondo e lo stato dei rapporti bilaterali tra i governi italiani e nigeriani durante la seconda metà degli anni Ottanta.

La ricerca effettuata ha consentito di far emergere un elemento particolarmente rilevante per quel che concerne la politica estera italiana e i rapporti con il governo

\footnotetext{
${ }^{84}$ Democrazia Proletaria, Dossier di “Pisa-Koko: il traffico dei rifiuti in Nigeria”, 14 luglio 1988, cit., pp. 17067-17073.

${ }^{85}$ Ivi, pp. 17079-17080.
} 
di Ibrahim Babangida negli anni Ottanta. Le criticità diplomatiche tra Italia e Nigeria, acuitesi nel corso dell'estate del 1988, si inserirono in un clima teso e "polemico", come ebbe a scrivere l'Istituto Affari Internazionali, in cui versavano tali relazioni almeno fin dall'estate del 1987. Un elemento che modifica lo scenario in cui si inserì la crisi del 1988, e che indica una strada per ulteriori e possibili sviluppi.

Con questo studio si è cercato di dare un contributo al dibattito storico sul tema dei traffici illeciti di rifiuti mettendo in luce sia la presenza di attori non necessariamente criminali - società di import-export, broker, avvocati, trasportatori - sia la portata politico-diplomatica dell'accaduto. Proprio per via delle criticità diplomatiche, è emerso come il tema si presti ad un'analisi di tipo storicointernazionalista e risulti altamente attuale sia per via della presenza di un problema sempre aperto, lo smaltimento dei rifiuti di provenienza industriale, sia per l'emergere, anno per anno, di fatti che hanno notevoli punti in comune con quanto accaduto sul finire degli anni Ottanta.

Occorre, quindi, prestare attenzione a quell'“economia sporca” a cui fa riferimento Vincenzo Ruggiero poiché è al suo interno che la richiesta di abbattimento dei costi di smaltimento delle industrie ha incontrato l'offerta di mafiosi, professionisti, broker, società di varia ragione sociale. ${ }^{86}$

\footnotetext{
${ }^{86}$ Vincenzo Ruggiero, Economie sporche. L'impresa criminale in Europa, Bollati Boringhieri, Torino, 1996; Vincenzo Ruggiero, "E' l'economia, stupido!" Una classificazione dei crimini di potere, in Sistemi criminali e metodo mafioso, Alessandra Dino, Livio Pepino (a cura di), Franco Angeli, Milano, 2008, pp. 188-208.
} 


\section{La ricerca}

\section{Bibliografia}

Albers Jan, Responsibility and Liability in the Context of Transboundary Movements of Hazardous Wastes by Sea, Springer, London, 2015.

Amadei Amedeo, Bottini Marino, Pecchenini Gabriella, (a cura di), Rischio rifiuti. Atti del convegno nazionale del 23 gennaio 1988 a Legnano, Centro Stampa Olgiati, Legnano, 1989.

Asante-Duah Kofi D., Imre V. Nagy, International Trade in Hazardous Waste, E\&FN Spon, New York, 1998.

Asante-Duah Kofi D., Saccomanno F. Frank, Shortreed John H., The Hazardous Waste Trade: can It be controlled?, in "Environmental, Science, Technology", Vol. 26, No. 9, 1992.

Bevilacqua Piero, La terra è finita. Breve storia dell'ambiente, Laterza, Roma-Bari, 2006.

Biliardo Ugo, Mureddu Giuseppe, Maree nere: rischio ambientale, in Il mosaico Mediterraneo, Santoro Carlo Maria (a cura di), Il Mulino, Bologna, 1991.

Bini Valerio, La cooperazione allo sviluppo in Africa. Teorie, politiche, pratiche, Mimesis, Milano-Udine, 2016.

Block Alan A., Environmental Crime and Pollution: Wasteful Reflections, in "Social Justice", Vol. 29, No. 1/2 (87-88), Globalization and Environmental Harm (2002).

Capria Antonella, Direttive ambientali CEE e stato di attuazione in Italia. Acqua, aria, rifiuti, in “Quaderni della rivista giuridica dell'ambiente”, v.1 n.1, 1992.

Carnì Andrea (a cura di), Cose storte. Documenti, fatti e memorie attorno alle "navi a perdere", Falco Editore, Cosenza, 2018.

Cianciullo Antonio, Fontana Enrico, Ecomafia. I predoni dell'ambiente, Editori Riuniti, Roma, 1995.

Clapp Jennifer, Toxic Exports: the transfer of hazardous wastes from rich to poor countries, Cornell University Press, Ithaca, 2001.

Clapp Jennifer, The Toxic Waste Trade with Less-Industrialised Countries: Economic Linkages and Political Alliances, in "Third World Quarterly", vol. 15, no. 3, 1994.

Corona Gabriella, Breve storia dell'ambiente in Italia, Il Mulino, Bologna, 2015.

Corona Gabriella, Sciarrone Rocco, Il paesaggio delle ecocamorre, in "Meridiana. Rivista di storia e scienze sociali", No. 43, Reti di mafie (2002). 


\section{La ricerca}

Dumont René, Démocratie pour l'Afrique, (1ºd. 1991), trad. It. Democrazia per l'Africa, Elèuthera, Milano, 1992.

Ebbe Obi N. I., Organized crime in Nigeria, in Traditional Organized Crime in the Modern World, Siegel Dina, van de Bunt H. (a cura di), New York, Springer Science \& Business Media, 2012.

Egidi Demetrio, Vannini Stefano, Le navi dei veleni e le bonifiche dei siti contaminati. Dalla gestione dell'emergenza una metodologia di intervento, Pitagora Editrice S.r.l., Bologna, 1995.

Ellis Stephen, This Present Darkness. A History of Nigerian Organised Crime, London, C. Hurst \& Co., 2016.

Emiliani Marcella, Petrolio, forze armate e democrazia. Il caso Nigeria, Carocci, Roma,2004.

Fabbri Fabrizio, Porto Marghera e la Laguna di Venezia. Vita, morte, miracoli, Jaca Book, Milano, 2003.

Falola Toyin, Paddock Adam, Environment and Economics in Nigeria, Routledge, Abingdon, 2013.

Garofalo Sabrina, Mobilitazione ambientale e anti-'ndrangheta di prossimità, in "Rivista di studi e ricerche sulla criminalità organizzata", vol. 3, n. 1, anno 2017.

George Susan, The Debt Boomerang, trad. it. Il boomerang del debito. Il debito del Terzo Mondo colpisce tutti, Edizioni Lavoro, Roma, 1992.

Germani Anna Rita, Pergolizzi Antonio, Reganati Filippo, Le determinanti del traffico organizzato di rifiuti in Italia: un'analisi empirica a livello regionale, in "Rivista economica del Mezzogiorno", Fascicolo 1-2, gennaio-giugno 2017.

Gilmore Lori, The Export of non-hazardous waste, in "Environmental Law" Vol. 19, No. 4 (Summer 1989).

Glickman Harvey, The Nigerian "419" Advance Fee Scams: Prank or Peril?, in "Canadian Journal of African Studies / Revue Canadienne des Études Africaines", Vol. 39, No. 3 (2005).

Greenpeace, The Toxic Ships. The Italian hub, the Mediterranean area and Africa, 2010.

Greenpeace, Waste Trade in The Mediterranean - Toxic Attack Against Lebanon Case One: Toxics From Italy, August 1996.

Hassan Zainab, Dumping on Somalia: A plea for environmental justice, "in Horn of Africa: an independent journal", v. 27, 2009.

Hilz Christoph, The International Toxic Waste Trade, Van Nostrand Reinhold, New York, 1992.

Istituto Affari Internazionali, L'Italia nella politica internazionale dal volume “1979-1980” al volume “1992-1993”. 


\section{La ricerca}

Ladapo Oluwafemi A., The Contribution of Cartoonists to Environmental Debates in Nigeria: The Koko Toxic-Waste-Dumping Incident, in "RCC Perspectives", n. 1, Eco-Images: Historical Views and Political Strategies, 2013.

Legambiente, Le nuove frontiere dell'ecomafia. Rapporto di Legambiente sull'illegalità ambientale in Italia e il ruolo della criminalità organizzata (1994-1997), 29 gennaio 1997.

Legambiente, I mercati globali dell'ecomafia, Palermo, dicembre 2000.

Lewis Peter, From Prebendalism to Predation: The Political Economy of Decline in Nigeria, in "The Journal of Modern African Studies", Vol. 34, No. 1 (Mar. 1996).

Liddick Donald R., Crimes Against Nature. Illegal Industries and the Global Environment, Praeger, Santa Barbara, 2011.

Liu Sylvia F., The Koko Incident: Developing International Norms for the Transboundary Movement of Hazardous Waste, in “Journal of Natural Resources \& Environmental Law", vol. 8, no. 1, 1992-1993. Lorenzini Sara, Una strana guerra fredda. Lo sviluppo e le relazioni Nord-Sud, Il Mulino, Bologna, 2017. Lorenzini Sara, Global Development. A Cold War History, Princeton University Press, Princeton, 2019. Lu Robert, West Africa: The Industrial World's Dumping Grounds, in "Harvard International Review", vol. 11, n. 4, Summer 1989.

Makdisi Karim S., Implementing International Environmental Treaties in Developing Countries: Using Flows to Explain How Lebanon Dealt with the Trade in Hazardous Waste, Paper Presented at the International Studies Association Annual Meeting 2006, San Diego, California, 23 March 2006.

Massari Monica, Paola Monzini, Dirty Businesses in Italy: A Case-study of Illegal Trafficking in Hazardous Waste, in "Global Crime”, Vol. 6 n. 3-4, August-November 2004.

McNeill John R., Something New Under the Sun. An Environmental History of the Twentieth-Century World, (2000) trad. It. Qualcosa di nuovo sotto il sole. Storia dell'ambiente nel XX secolo, Einaudi, Torino, 2002.

Melandri Giovanna (a cura di), Ambiente Italia. Rapporto 1989: dati, tendenze, proposte, Isedi Petrini Editore, Torino, 1989.

Melandri Giovanna, Conte Giulio (a cura di), Ambiente Italia 1991, Milano, Mondadori, 1991.

Montgomery Mark A., Reassessing the Waste Trade Crisis: What Do We Really Know?, in "The Journal of Environment \& Development", Vol. 4, No. 1 (Winter 1995).

Moyers Bill, Global Dumping Ground. The International Traffic in Hazardous Waste, The Lutterworth Press, Cambridge, 1991. 


\section{La ricerca}

Muawya Ahmed Hussein, Costs of environmental degradation: An analysis in the Middle East and North Africa region, in "Management of Environmental Quality. An International Journal", vol. 9, n. 3, anno 2008.

Müller Simone M., Hidden Externalities: The Globalization of Hazardous Waste, in "Business History Review", n. 93 (spring 2019).

Noah Monday Efiong, Inland Ports and European Trading Firms in Southeastern Nigeria, in "African Affairs", Vol. 88, No. 350 (Jan., 1989).

0’Keefe Phil, Toxic Terrorism, in "Review of African Political Economy", n. 42 (1988).

Obi Cyril, Globalization and Environmental Conflict in Africa, in "African Journal of Political Science / Revue Africaine de Science Politique", Vol. 4, No. 1 (June 1999).

Oluwafemi A. Ladapo, The Contribution of Cartoonists to Environmental Debates in Nigeria: The Koko Toxic-Waste-Dumping Incident, in "RCC Perspectives", n. 1, Eco-Images: Historical Views and Political Strategies, 2013.

Osservatorio Permanente su Ambiente e Legalità, Le "ecomafie". Il ruolo della criminalità organizzata nell'illegalità ambientale, Roma, dicembre 1994.

Palladino Andrea, Bandiera nera. Le navi dei veleni, Manifestolibri, Roma, 2010.

Pellegrini Stefania, L'impresa grigia. Le infiltrazioni mafiose nell'economia legale, Ediesse, Roma, 2019.

Rebovich Donald J., Dangerous Ground. The World of Hazardous Waste Crime, Transaction Publishers, New Brunswick, 1992.

Roelants Du Vivier François, Les vaisseaux du poison, Editions Sang de la terre, Paris, 1988.

Royal Institute of International Affairs, Sustainable Development Programme, International Environmental Crime. The Nature and Control of Environmental Black Markets, Background paper for RIIA workshop, 27-28 May 2002 di Duncan Brack, Head of Programme e Gavin Hayman, Associate Fellow.

Ruggiero Vincenzo, Economie sporche. L'impresa criminale in Europa, Bollati Boringhieri, Torino, 1996.

Sales Isaia, Melorio Simona, Storia dell'Italia corrotta, Rubbettino, Soveria Mannelli, 2019.

Sales Isaia, La questione rifiuti e la camorra, in "Meridiana. Rivista di storie e scienza sociali", n. 7374, anno 2012. 


\section{La ricerca}

Scovazzi Tullio, Immersione di sostanze inquinanti in mare e risarcimento del danno, in "Rivista giuridica dell'ambiente", fascicolo 1, 1986.

Tolba Mostafa K., El-Kholy Osama, The World Environment 1972-1992. Two decades of challenge, Springer Science\&Business Media, Oxford, 1992.

Uwagbale Edward-Ekpu Douglas, Hazardous Waste Management and Challenges in Nigeria, in "Public Health International”, 1 (1), 2016.

Vallette Jim, Spalding Heather (a cura di), The International Trade in Wastes: A Greenpeace Inventory, Greenpeace International Waste Trade Project, Washington DC, 1990.

Van Daele Stijn, Vander Beken Tom, Dorn Nicholas, Waste Management and Crime - Regulatory, Business and Product Vulnerabilities, in "Environmental Policy and Law", vol. 37, n.1 (2007).

Van Solinge Tim Boekhout, The illegal exploitation of natural resources, in The Oxford Handbook of Organized Crime, Letizia Paoli (a cura di), Oxford University Press, New York, 2014.

White Rob, Toxic Cities: Globalizing the Problem of Waste, in Social Justice, Vol. 35, No. 3 (113), War, Crisis \& Transition (2008-09).

White Rob, Transnational Environmental Crime. Toward an eco-global criminology, Routledge, Abingdon, 2011.

White Rob, The Conceptual Contours of Green Criminology, in Emerging Issues in Green Criminology, Walters Reace, Solomon Westerhuis Diane, Wyatt Tanya (a cura di), Palgrave Macmillan, New York, 2013.

Wyatt Tanya (a cura di), Hazardous Waste and Pollution. Detecting and Preventing Green Crimes, Springer, London, 2016.

Zumerchik John, Danver Steven L., Seas and Waterways of the World: An Encyclopedia of History, Uses, and Issues, Vol. 1, ABC-Clio, Santa Barbara (California), 2008.

\section{Fonti orali}

Giorgio Bascherini, marinaio Piave, intervista realizzata da Bruno Luverà per "Radio Radicale", 2 agosto 1988: https://www.radioradicale.it/scheda/28253/scorie-radioattive-italiane-in-nigerialiberata-la-nave-piave-bloccata-per-ritorsione.

\section{Fonti edite}

Commissione parlamentare d'inchiesta sul ciclo dei rifiuti, XVI legislatura, Audizione di Renato Pent, Bologna, 17 febbraio 2010. 


\section{La ricerca}

Commissione parlamentare d'inchiesta sul ciclo dei rifiuti, XVII legislatura, Relazione sulle «navi dei veleni». I traffici internazionali di rifiuti negli anni ' 80 e '90, doc. XXIII N. 51, approvata il 28 febbraio 2018.

Ministero degli Affari Esteri, Testi e documenti sulla politica estera dell'Italia, volumi dall'anno 1980 al 1993.

Ministro per il Coordinamento della Protezione Civile Vito Lattanzio, Ordinanza del 21 settembre 1988 del (Ordinanza n. 1561/FPC), "Ulteriori disposizioni eccezionali afferenti alle operazioni finalizzate allo smaltimento definitivo delle sostanze tossiche e nocive trasportate dalla nave Karin B" (GU Serie Generale n.231 del 01-10-1988).

Organisation for Economic Co-Operation and Development, Environmental Indicators, Paris, OECD, 1991.

Senato della Repubblica, X Legislatura, Disegno di legge n. 969 d'iniziativa dei senatori Azzaretti, Angeloni e Meriggi (DC-PCI) comunicato alla presidenza il 7 aprile 1988 sulle "Misure per garantire il pagamento dei crediti vantati da imprese italiane nei confronti di clienti nigeriani". https://www.senato.it/service/PDF/PDFServer/DF/267934.pdf.

Senato della Repubblica, XIII Legislatura, Disegno di legge n. 4499 d'iniziativa dei senatori Servello, Basini, Magliocchetti, Turini, Demasi e Pontone comunicato alla presidenza il 24 febbraio 2000 in merito alla "Concessione di un equo indennizzo ad imprese italiane e cittadini italiani che hanno subito perdite patrimoniali in http://www.senato.it/japp/bgt/showdoc/13/Ddlpres/0/00005757/index.html?part=doc_dc.

Senato della Repubblica, XIII legislatura, $163^{\circ}$ seduta (pomeridiana), 3 aprile 1997, Resoconto Stenografico, Interrogazione parlamentare (n. 4-05147) dei senatori Montagna e Marchetti al Presidente del Consiglio dei ministri e ai Ministri degli affari esteri e per gli italiani all'estero e di grazia e giustizia.

United Nations, Economic and Social Council 40 th plenary meeting, 28 july 1988 (1988/70), Traffic in toxic and dangerous products and wastes.

United Nations, General Assembly, 44th session, 18 July 1989 (A/44/362), Development and International Economic Co-Operation: Environment. Illegal traffic in toxic and dangerous products and wastes.

United Nations, Security Council, 66 $6^{\text {th }}$ session, 25 October 2011, Report of the Secretary-General on the protection of Somali natural resources and waters.

World Bank, World Debt Tables: external debt of developing countries (1991-1992), Washington, 1991.

World Resources Institute, World Resources, 1990-91, New York, Basic Books, 1991. 


\section{La ricerca}

\section{Fonti inedite}

Archivio Centrale dello Stato, Roma:

- Fondo della Presidenza del Consiglio dei Ministri (1860-2000), Ufficio del Consigliere Diplomatico, II parte (1964-1985).

- $\quad$ Fondo Italia Nostra 1955-1992.

- $\quad$ Fondo Partito della Rifondazione Comunista, Direzione nazionale 1991-2010.

- $\quad$ Fondo Gruppo Parlamentare Sinistra indipendente, Camera 1987-1992.

- $\quad$ Fondo del Ministero dell'Interno, Dipartimento Pubblica Sicurezza (dal 1981), Segreteria del Dipartimento, Ufficio ordine pubblico, Categorie varie 1946-1992.

Archivio Storico della Camera dei Deputati, Roma:

- Fondo Commissioni parlamentari d'inchiesta sul ciclo dei rifiuti e sulle attività illecite ad esso connesse (1995-2018).

Archivio della Procura di Asti:

- $\quad$ Procedimento penale n. 395/97.

Archivio della Procura di Paola:

- $\quad$ Procedimento penale n. 5085/03.

Archivio generale del Tribunale di Milano:

- $\quad$ Procedimento civile n. 11117/95.

- $\quad$ Procedimento civile n. 76616/2004. 